\title{
VIRTUAL OUTPATIENTS
}

\author{
S Hulikere ${ }^{1}$, A Gopalakrishna² \& E Thomason ${ }^{3}$ \\ ${ }^{1}$ Consultant Paediatrcian and Clinical Director, Women's and Children's Health, \\ Warrington and Halton Teaching Hospitals NHS Foundation Trust \\ ${ }^{2}$ Consultant Paediatrician, Warrington and Halton Teaching Hospitals NHS \\ Foundation Trust \\ ${ }^{3}$ ST8 Paediatric Trainee in the North West
}

\section{STAYING CONNECTED WITH PATIENTS - THE NEW NORMAL IN THE OUTPATIENT MANAGEMENT OF CHILDREN:}

The NHS has faced many challenges in the past, but none quite as unprecedented or as unique as COVID-19. The COVID-19 pandemic has led to rapid NHS procedure changes out of necessity; it has tested the NHS's limits and its capacity. That being said, it has also provided lots of opportunities and food for thought regarding future work and methods to deliver care. One of these areas is the outpatient management of children referred to secondary care.

In 2018/19, there were over 123 million outpatient appointments at NHS hospitals in England, but 22\% of the referrals were either not attended or were cancelled. ${ }^{1}$ A quarter of doctors that participated in a survey by the Royal College of Physicians felt that 10$20 \%$ of their new patients did not need an appointment at all. ${ }^{2}$ The NHS Long Term Plan aims to reduce the number of outpatient attendances by up to a third over the next 5 years. ${ }^{3}$

\section{WHAT HAS HAPPENED TO CHILDREN NEEDING TO BE SEEN IN OUTPATIENTS DURING COVID-19?}

Many trusts have had to cancel NHS outpatient work during the COVID-19 pandemic to create enough capacity in the system to meet the rising number of cases. Significant numbers of patients also cancelled or did not attend outpatient appointments during the COVID-19 pandemic due to concerns around the risk of attending hospitals.

Clinicians were asked to triage patients from their clinic list and defer patients appropriately based on an individual risk assessment. Those who were scored highly (or in the "red" category) were provided appointments, but those classified as lower risk (or 
"green") were placed on an access plan for the next available appointment. However, during the same period, the referral to outpatients from both GPs and secondary care reduced as fewer patients utilised the system. At Warrington and Halton Teaching Hospitals (WHH), there were 1472 outpatient referrals in the 0-18 year age group for the first financial quarter, compared to 4514 in the same period of 2019. This is a reduction of total referrals by $68 \%$, and a reduction in routine referrals by $86 \%$. Since the Government has eased restrictions, GPs have begun reviewing cases and referrals are gradually increasing towards prior levels.

The combination of cancelled clinics, deferred patients, data quality issues, more patients accessing health care post-stage 4 lockdown, and increasing GP referrals to secondary care has led to incredible pressure on outpatients due to lack of capacity.

\section{VIRTUAL CLINIC APPOINTMENTS:}

COVID-19 has accelerated the implementation of virtual clinics within the NHS. A virtual clinic complements face-toface consultations, as not all patients are suitable for virtual clinics. Identifying the patients that are suitable for virtual consultation is therefore an important first step.

A remote consultation is an appointment between a patient and a clinician over the telephone or video. Telephone consultations are not new; many specialist paediatric nurses have used them in areas such as epilepsy, diabetes, neonatal, and as well as by community teams. They have benefitted the NHS by reducing emergency department attendance and hospital admissions. Studies have suggested that up to $38 \%$ of in-person appointments could be avoided in some specialties with no negative impact on the clinical outcomes. ${ }^{4}$ The systems needed to undertake telephone consultations exist in primary care and most secondary care providers. Telephone consultations are easy to adopt and have thus been a success in many trusts. ${ }^{5}$

Most clinics in the Paediatrics department of Warrington and Halton Teaching Hospitals were converted to telephone clinics following the initial UK COVID-19 restrictions in March. The telephone consultations have not only supported the continuity of care but provided aid to many families who are understandably anxious about coming to the hospital at this time. Alleviating this particular anxiety may have also curbed the number of missed appointments. Additionally, telephone consultation reduces travel costs and time for patients and alleviates most trusts' car parking issues.

Many trusts are piloting video consultations and implementing them on a wide scale. This new way of working and the quick uptake by providers and patients offers new opportunities moving forwards in a post-COVID-19 world. The number of clinicians working from home and remotely conducting the clinics has increased due to the pandemic. However, there are challenges with home working, clinicians report frustrations with technical problems with NHS laptops. Personal laptops cannot be used for consultations for data security reasons.

Studies have found that patients are amenable to healthcare delivery via telemedicine in various circumstances, ${ }^{6}$ although care delivery should be individualised to the patient's needs. During the COVID-19 pandemic, general paediatric follow-ups and specialised clinics like allergies, diabetes, and epilepsy were delivered virtually at WHH. Most virtual clinics reported good parent engagement. In June 2020, one consultant held 4 diabetes clinics and arranged 31 telephone appointments. There was $100 \%$ attendance, whereas face-to-face diabetes clinics in 2019-2020 had a Did Not Attend (DNA) rate of 5.7\%. For general paediatric clinics, a review of telephone followups undertaken between April-June 2020 had a DNA rate of $12 \%$, which is similar to DNA rates before COVID-19. Of note, there were no DNAs to the clinic in April, which may be because telephone and text reminders were sent as part of the transition to virtual clinics, but this was only done in April. Therefore, it is evident that virtual clinics can reduce DNA rates when appropriate systems are in place to aid patient engagement. If such measures are not taken, then virtual clinics will not achieve the NHS long term plan. As non face-toface clinics are a relatively new model of care, the trusts should carefully look at the impact of virtual clinics and demonstrate efficiency by appropriate evaluations.

Not all patients and cases are suitable for virtual consultations. During COVID-19, all new patient referrals to services were delivered face-to-face in the paediatric department of $\mathrm{WHH}$. The patients in cardiology clinics were also seen face to face as they required diagnostic investigations such as echocardiograms, but the clinics were delivered with a reduced capacity. There is no one-size-fits-all approach to drafting the hybrid clinic templates for the new outpatient services model. The templates must be tailored to the clinic type, and this is best achieved through greater engagement 
with clinicians and patients. The new outpatient care models will be evaluated through testing with PDSA (Plan, Do, Study, Act) cycles to improve service delivery and take steps towards meeting the NHS Long Term Plan.

While there are undoubted savings in costs, the biggest gain in "value" is from the robustness and quality of the process, and the chance to involve a greater proportion of the population.

\section{SUPERVISION AND TEACHING TRAINEES:}

Teaching trainees in outpatient settings can often be difficult due to service demand; there is no right way to support their learning, as the settings are often opportunistic. There is a need for cultural change to ensure trainees' competence and support their educational needs with new demands on their communication skills within the 'new normal'. COVID-19 has prompted WHH to implement independent practice for senior trainees and direct Consultant supervision of junior trainees. This was largely successful; however, there is certainly a need for further training of health care professionals regarding teaching in virtual clinics.

\section{WHAT IF WE NEED FACE-TO- FACE APPOINTMENTS?}

Not all patients are suitable for virtual clinics, and face-toface clinic appointments provide advantages to clinicians. For example, assessing patients with potentially serious, highrisk conditions cannot be deferred if they are likely to need a physical examination. Face-to-face appointments are also used for patients with no access to the technology and those unable to communicate over telephone or video (e.g., deaf or hard-of-hearing patients). Moreover, virtual clinics are also not suitable when there are safeguarding concerns. National Guidance and individual clinical teams' approval have resulted in a gradual reintroduction of face-to-face appointments across the UK. Trusts have adopted new hybrid clinic templates and robust triaging to support social distancing guidelines.

In order to support social distancing, waiting room capacity must be accounted for. It is important to ensure that outpatient department teams are geared up to greet patients, manage visitors, and maintain flow to avoid overcrowding. Some trusts have dedicated limited parking near outpatients to support extending the waiting areas. The clinicians have also created drive through appointments for quicker face-toface appointments, largely for routine services such as blood tests, swabbing and immunisations. Clinicians need to be on time and appropriately manage patient flow. Identification of shielding clinic rooms supported by clear operating procedures and designated parking for shielding patients on the day of appointment must be considered.

\section{HOW TO INCREASE THE OUTPATIENT CAPACITY AND ENSURE THE SAFETY OF OUR PATIENTS?}

The implementation of telephone and video clinics has aided in providing care and managing outpatients. It has supported patients who cannot travel to hospitals, such as those in atrisk groups or self-isolating. During the recovery phase, face to face consultations have increased, but are still limited due to the required social distancing measures. This has increased the time until patients are seen following a referral.

Self-isolating staff and those in at-risk groups have been able to support providers to meet increased demand. Nevertheless, they are reliant on secondary care providers to supply facilities for accessing patient records. Not all trusts are prepared for this new remote working but are supporting clinicians where possible.

Retired returners have helped to increase the capacity of clinics in the short term. Longer contracts with appropriate revalidation of their registration need to be considered due to increasing waiting lists because of COVID-19 disruptions.

Going forward, trial measures such as weekend working may improve the capacity and ensure patients are seen in an appropriate timeframe. 


\section{OUTPATIENT RECOVERY PLAN - WHAT DOES IT LOOK LIKE?}

The COVID-19 pandemic has created a series of challenges and provided many opportunities for new working methods. The 'new normal' services heavily rely on technology. It is up to individual trusts to embrace technology and adopt new methods quickly to ensure continuity of patient care. They must recognise and respond to the 'new normal' rather than maintaining past practice.

Clinical triaging is a focal area in this new system. NHS England has issued advice and guidance that has been implemented in recent years and may reduce inappropriate referrals.

Delivering virtual clinics and establishing appropriate templates are important, but these require clinician and patient engagement. Outpatient management teams must work closely with clinicians of all specialities to make appropriate practice templates to reflect face-to-face and telephone clinic appointments.

NHS England and NHS Improvement's support is offered to secondary care providers, most of whom are already set up to carry out phone consultations. To help accelerate the uptake throughout the pandemic, NHS England and NHS Improvement have procured 12-month licences for the video consultation platform Attend Anywhere. These licences will be available free of charge to all NHS secondary care providers. The proposed 2020/21 tariff sets out that non-face-to-face activity must have the same unit price as a face-to-face attendance.

\section{IMPROVING EFFICIENCY THROUGH VIRTUAL OUTPATIENT CLINICS:}

The traditional outpatient model is not fit for purpose and is outdated. 1 in 5 clinic appointments are either not attended or cancelled. ${ }^{1}$ Moreover, repeated patient transport takes up a considerable amount of time and increases the individual's carbon footprint. Analysis in the West Midlands estimated f5.34 GVA (gross value added, an economic productivity impact) annually if $10 \%$ of outpatient follow-ups converted to virtual clinic appointments. ${ }^{7}$ The analysis also estimated a 2.5-minute saving per appointment, which has the potential to make available an additional 5,200 hours of appointment time. Additionally, from a patient point of view, the projected cost savings ranged from $£ 325 \mathrm{k}$ - $£ 973 \mathrm{k}$ (excluding lost income) per annum. Reduced travel costs had potential benefits on the environment, between 177,845 to $533,535 \mathrm{~kg}$ CO2 annually through reduced travel. ${ }^{7}$

Delivering virtually also improves efficiency by increasing clinic capacity for the trusts. This was successfully demonstrated during COVID-19 at WHH; despite limited estate capacity due to social distancing, all paediatric clinics were delivered from one site instead of the usual two. This has motivated the management team to base all future outpatient services for children at one site. It shows that virtual clinics can benefit not only patients but also clinicians, management, service providers, commissioners, the environment, and the overall health economy.

\section{FUTURE PRACTICE:}

The COVID-19 pandemic has instigated huge changes in outpatient working practices through increased telephone consultations and the gradual introduction of video consultations in the trusts. Most feel the consultations are generally quicker; however, specific patient issues and a clinician's ability to conduct virtual assessments are still areas to monitor. Telephone consulting requires considerable skill and judgement due to the lack of visual cues. ${ }^{8}$

Some children with suspected COVID-19 or who are in isolation need case-specific accommodations and support for clinic appointments. A short-term solution is the deferment of appointments until a face-to-face consultation becomes safe. However, establishing mobile drive-through clinics or dedicated 'hot' clinics in outpatients may also be potential solutions, as COVID-19 is set to remain a major issue for the foreseeable future.

One of the NHS's founding principles is striving to deliver the best care for the people who rely on NHS services. The COVID-19 pandemic has certainly changed how we implement outpatient services and deliver care in secondary care, but it has not shaken this core belief. We do not know what the future may hold, but it appears that a hybrid of virtual and face to face appointments is the current best practice for the 
future management of outpatient referrals to secondary care. While COVID-19 is a disease that knows no boundaries and has already had a devastating impact, it has also created an environment for real transformation and redesign, making outpatient services fit for the future.

\section{KEY WORDS:}

COVID-19 pandemic, remote consultation, telephone clinics, video consultation, clinic capacity, outpatient recovery.

\section{REFERENCES:}

1. NHS Digital. Hospital Outpatient Activity [Internet]. 2019 Oct. Available from: https://files.digital.nhs.uk/33/ EF9007/hosp-epis-stat-outp-summ-rep-2018-19-rep.pdf

2. Royal College of Physicians. Outpatients: the futureadding value through sustainability. RCP; 2018.

3. NHS England. The Long-Term Plan [Internet]. 2019 p. 25-7. Available from: https://www.longtermplan.nhs.uk/

4. Car J. Telephone consultations. BMJ. 2003 May 3;326(7396):966-9. http://doi.org/10.1136/ bmj.326.7396.966

5. Greenhalgh T, Vijayaraghavan S, Wherton J, Shaw S, Byrne E, Campbell-Richards D, et al. Virtual online consultations: advantages and limitations (VOCAL) study. BMJ Open. 2016 Jan;6(1):e009388. http://doi. org/10.1136/bmjopen-2015-009388

6. Mair F. Systematic review of studies of patient satisfaction with telemedicine. BMJ. 2000 Jun 3;320(7248):1517-20. http://doi.org/10.1136/ bmj.320.7248.1517

7. The Strategy Unit. The Potential Economic Impact of Virtual Outpatient Appointments in the West MidlandsA scoping study [Internet]. NHS Midlands and Lancashire Commissioning Support Unit; 2018 Oct. Available from: https://www.strategyunitwm.nhs.uk/publications/ potential-economic-impact-virtual-outpatientappointments-west-midlands-scoping-study
8. Greenhalgh T, Wherton J, Shaw S, Morrison C. Video consultations for COVID-19. BMJ. 2020 Mar 12;m998. http://doi.org/10.1136/bmj.m998

\section{.}

